\title{
High temperature asymptotics of thermodynamic functions of electromagnetic field subjected to boundary conditions on a sphere and cylinder
}

\author{
M. Bordag* \\ Universität Leipzig, Institut für Theoretische Physik \\ Augustusplatz 10, 04109 Leipzig, Germany \\ V.V. Nesterenko ${ }^{\dagger}$, I.G. Pirozhenko \\ Bogoliubov Laboratory of Theoretical Physics \\ Joint Institute for Nuclear Research, Dubna, 141980, Russia
}

(November 1, 2018)

\begin{abstract}
The high temperature asymptotics of thermodynamic functions of electromagnetic field subjected to boundary conditions with spherical and cylindrical symmetries are constructed by making use of a general expansion in terms of heat kernel coefficients and the related determinant. For this, some new heat kernel coefficients and determinants had to be calculated for the boundary conditions under consideration. The obtained results reproduce all the asymptotics derived by other methods in the problems at hand and involve a few new terms in the high temperature expansions. An obvious merit of this approach is its universality and applicability to any boundary value problem correctly formulated.
\end{abstract}

12.20.Ds, 03.70.+k, 78.60.Mq, 42.50.Lc

Typeset using REVTEX

*E-mail: Michael.Bordag@itp.uni-leipzig.de

${ }^{\dagger}$ E-mail: nestr@thsun1.jinr.ru

${ }^{\ddagger}$ E-mail: pirozhen@thsun1.jinr.ru 


\section{INTRODUCTION}

The Casimir effect is one of the most interesting phenomena in quantum field theory. Since its discovery more than 50 years ago it attracted much attention. In the past years the interest intensified after its experimental verification reached the one percent level of precision [1] [3].

The influence of temperature on the Casimir effect was an important topic since its first experimental demonstration [4] which had been done at room temperature. It was first shown in Ref. [5] that the temperature influence was just below what had been measured. It is expected that the temperature contributions will be seen in the upcoming series of experiments.

In quantum field theory, finite temperature effects can be described at equilibrium in the Matsubara formalism by imposing periodic (resp. antiperiodic for fermions) boundary conditions in the imaginary time coordinate. Technically this is very similar to the calculation of the Casimir effect for plane boundaries and can mathematically be described by the same Riemann and Hurwitz zeta functions. Another formula important for applications is the well known Lifshitz formula describing the interaction between plane dielectric bodies at finite temperature. In the case of nonflat boundaries the situation is, however, more complicated. In order to obtain the Casimir effect at finite temperature one has to know it at least at zero temperature, i.e., one has to know the spectrum of the corresponding operator. Even then complicated calculations are usually necessary and explicit results are rare.

An opposite situation occurs with the asymptotic expansion of the Casimir energy at high temperature. It turns out to be determined to a large extent by local quantities which are much easier to obtain. In the paper [6], which did not receive the due attention, it was shown that this expansion can be written down in terms of the heat kernel coefficients and the functional determinant of the operator corresponding to the spatial part of the problem.

For example, this method has been applied in [7 9] for the effective potential in curved spacetimes and for the Casimir effect for hypercuboids.

The heat kernel coefficients for differential operators on manifolds with and without boundary are known to depend on the properties of this manifold only locally. This means that they can be represented as integrals over the manifold and over its boundary [10] whereby the characteristics like curvature enter as local functions. The calculation of these coefficients is a topic of its own and much progress had been achieved especially during the past decade (see the book [11], for example). Less is known about the determinant. It is not ascertain in general whether it is a local quantity. However, in several examples it is shown to be calculable much easier than the corresponding Casimir energy at zero temperature. As a consequence, the high temperature expansion of the Casimir energy can be calculated quite easily. This was emphasized in the recent review [12] where the basic formula (2.10) is taken from.

In the present paper we apply these general formulas to some specific examples. First we consider parallel plates in order to demonstrate the technical tools on a simple problem. Then we consider the conducting spherical and cylindrical shells obtaining new terms in the asymptotic expansion. Eventually we consider the dielectric ball and cylinder where we restrict ourselves to the dilute approximation. These examples demonstrate the effectiveness of the method. 
An interesting application of the general formula is the discussion of the so called classical limit which had recently been considered in Ref. [13]. It is understood as to take place if the internal energy which is connected with the free energy by Eq. (2.11) (see below the next section) tends to zero for $T \rightarrow \infty$. This happens if the heat kernel coefficients with number $n \leq \frac{3}{2}$ vanish.

The first calculation of the leading contributions to the high temperature asymptotics of the Casimir energy for curved boundaries was given in Ref. [14 using the multiple reflection expansion. As it was noticed in the recent paper [15] the multiple reflection expansion can be used for the calculation of the heat kernel coefficients demonstrating the equivalence of both approaches up to the question of the determinant.

The situation is to some extent different for boundaries with edges and corners. Here the application of Riemann and Hurwitz zeta functions seems to be more appropriate. A first example of this kind was given in Ref. [16]. The appropriate more general methods can be expected to be those given in Ref. [17].

The layout of the paper is as follows. In Sec. II the derivation of the high temperature expansions in terms of the heat kernel coefficients is briefly given. In Sec. III the original setting of the Casimir effect, i.e. parallel perfectly conducting plates in vacuum, is considered and the high temperature asymptotics of the thermodynamic functions are derived in terms of the relevant heat kernel coefficients. In Sec. IV the high temperature asymptotics for electromagnetic field with boundary conditions on a sphere are obtained. In Sec. V the high temperature expansions are constructed for the boundary conditions defined on the lateral of a circular infinite cylinder. The heat kernel coefficients needed are calculated by making use of the respective zeta functions that have been obtained in an explicit form in terms of the Riemann zeta function in Ref. [18] and also by applying the results of Ref. [19]. The functional determinants entering the asymptotic expansions at hand are calculated by making use of the technique developed in Ref. [20]. The results obtained are compared with the high temperature asymptotics which have been derived for boundary conditions under consideration by other methods. The possible extension of the approach is discussed in the Conclusions (Sec. VI).

The mathematical details of the calculation of the zeta determinants are presented in Appendix A for electromagnetic field subjected to boundary conditions given on a sphere and in Appendix B for the boundary conditions defined on the lateral of an infinite circular cylinder.

\section{HEAT KERNEL COEFFICIENTS AND HIGH TEMPERATURE EXPANSIONS}

Let the dynamics of quantum field be defined by the operator

$$
\frac{1}{c^{2}} \frac{\partial^{2}}{\partial t^{2}}-\Delta
$$

where $\Delta$ is not of necessity the Laplace operator, but an elliptic differential operator depending only on space coordinates. The free energy $F$ of the field is determined by the zeta function $\zeta_{\mathrm{T}}(s)$ corresponding to the Euclidean version of the operator (2.1) 


$$
F=-\frac{T}{2} \zeta_{\mathrm{T}}^{\prime}(0)
$$

Here $T$ is the temperature measured in energy units (the Boltzmann constant $k_{\mathrm{B}}$ is assumed to be equal to 1$)$, and the zeta function $\zeta_{\mathrm{T}}(s)$ is defined in a standard way

$$
\zeta_{\mathrm{T}}(s)=\sum_{m=-\infty}^{\infty} \sum_{\{k\}}\left(\Omega_{m}^{2}+\omega_{k}^{2}\right)^{-s}
$$

with $\Omega_{m}=2 \pi m T / \hbar$ being the Matsubara frequencies and $\omega_{k}^{2} / c^{2}$ standing for the eigenvalues of the operator $-\Delta$ in Eq. (2.1)

$$
-\Delta \varphi_{k}(\boldsymbol{x})=\frac{\omega_{k}^{2}}{c^{2}} \varphi_{k}(\boldsymbol{x})
$$

The characteristics of the quantum field system with dynamical operator (2.1) at zero temperature are determined by the zeta function $\zeta(s)$ associated with the operator $-\Delta$

$$
\zeta(s)=\sum_{\{k\}} \omega_{k}^{-2 s}
$$

From the mathematical point of view the zeta function $\zeta(s)$ corresponding to the space part of the operator (2.1) is, undoubtedly, a simpler object than the complete zeta function $\zeta_{\mathrm{T}}(s)$ because the definition (2.3) involves an additional sum over the Matsubara frequencies. Here a natural question arises whether one can gain knowledge of the quantum field at nonzero temperature possessing only the zeta function $\zeta(s)$. In Ref. [6] it was shown that proceeding from the zeta function $\zeta(s)$ one can deduce the high temperature asymptotics of the thermodynamic functions such as Helmholtz free energy, internal energy, and entropy. Let us remind briefly the derivation of these asymptotics. By making use of the formula

$$
\lambda^{-s}=\frac{1}{\Gamma(s)} \int_{0}^{\infty} d t t^{s-1} e^{-\lambda t}
$$

the zeta function (2.3) can be represented in the form

$$
\zeta_{\mathrm{T}}(s)=\frac{1}{\Gamma(s)} \int_{0}^{\infty} d t t^{s-1} \sum_{m=-\infty}^{\infty} e^{-\Omega_{m}^{2} t} \sum_{\{k\}} e^{-\omega_{k}^{2} t} .
$$

The term with $m=0$ in this formula gives the zeta function (2.5). In the remaining terms we substitute the heat kernel $K(t)$ of the operator $-\Delta$ by its asymptotic expansion at small $t$

$$
K(t) \equiv \sum_{\{k\}} e^{-\omega_{k}^{2} t} \simeq \frac{1}{(4 \pi t)^{3 / 2}} \sum_{n=0,1 / 2, \ldots} a_{n} t^{n}+\ldots
$$

As a result we arrive at the following asymptotic representation for the complete zeta function $\zeta_{\mathrm{T}}(s)$

$$
\zeta_{\mathrm{T}}(s) \simeq \zeta(s)+\frac{2}{(2 \pi)^{3 / 2}} \sum_{n=0,1 / 2, \ldots} a_{n}\left(\frac{\hbar}{2 \pi T}\right)^{2 s-3+2 n} \frac{\Gamma(s-3 / 2+n)}{\Gamma(s)} \zeta_{\mathrm{R}}(2 s+2 n-3),
$$


where $\zeta_{\mathrm{R}}(s)$ is the Riemann zeta function. Taking the derivative of the right hand side of Eq. (2.9) at the point $s=0$ and substituting the result into Eq. (2.2) one obtains the high temperature expansion for the free energy

$$
\begin{aligned}
F(T) \simeq & -\frac{T}{2} \zeta^{\prime}(0)+a_{0} \frac{T^{4}}{\hbar^{3}} \frac{\pi^{2}}{90}-a_{1 / 2} \frac{T^{3}}{4 \pi^{3 / 2} \hbar^{2}} \zeta_{\mathrm{R}}(3)-\frac{a_{1}}{24} \frac{T^{2}}{\hbar}+\frac{a_{3 / 2}}{(4 \pi)^{3 / 2}} T \ln \frac{\hbar}{T} \\
& -\frac{a_{2}}{16 \pi^{2}} \hbar\left[\ln \left(\frac{\hbar}{4 \pi T}\right)+\gamma\right]-\frac{a_{5 / 2}}{(4 \pi)^{3 / 2}} \frac{\hbar^{2}}{24 T} \\
& -T \sum_{n \geq 3} \frac{a_{n}}{(4 \pi)^{3 / 2}}\left(\frac{\hbar}{2 \pi T}\right)^{2 n-3} \Gamma(n-3 / 2) \zeta_{\mathrm{R}}(2 n-3), \quad T \rightarrow \infty .
\end{aligned}
$$

Here $\gamma$ is the Euler constant. The argument of the logarithms in expansion (2.10) are dimensional, but upon collecting similar terms with account for the logarithmic ones in $\zeta^{\prime}(0)$ it is easy to see that finally the logarithm function has a dimensionless argument, at least for $a_{2}=0$. Let us note that according to the definition (2.8) the heat kernel coefficients in our consideration are dimensional, because the frequencies $\omega_{k}$ have the dimensionality [time] ${ }^{-1}$.

The asymptotic expansions for the internal energy $U(T)$ and the entropy $S(T)$ are deduced from Eq. (2.10) employing the thermodynamic relations

$$
\begin{aligned}
& U(T)=-T^{2} \frac{\partial}{\partial T}\left(T^{-1} F(T)\right), \\
& S(T)=T^{-1}(U(T)-F(T))=-\frac{\partial F}{\partial T} .
\end{aligned}
$$

Substituting the expansion (2.10) into Eqs. (2.11) and (2.12) one arrives at the asymptotics

$$
\begin{aligned}
U(T) \simeq & a_{0} \frac{T^{4}}{\hbar^{3}} \frac{\pi^{2}}{30}+a_{1 / 2} \frac{T^{3}}{\hbar^{2}} \frac{\zeta_{\mathrm{R}}(3)}{2 \pi^{3 / 2}}+a_{1} \frac{T^{2}}{24 \hbar}+\frac{a_{3 / 2}}{(4 \pi)^{3 / 2}} T \\
& -a_{2} \frac{\hbar}{16 \pi^{2}}\left[\ln \left(\frac{\hbar}{4 \pi T}\right)+\gamma+1\right]-\frac{a_{5 / 2}}{(4 \pi)^{3 / 2}} \frac{\hbar^{2}}{12 T} \\
& -\frac{T}{4 \pi^{3 / 2}} \sum_{n \geq 3} a_{n}\left(\frac{\hbar}{2 \pi T}\right)^{2 n-3} \Gamma(n-1 / 2) \zeta_{\mathrm{R}}(2 n-3), \\
S(T) \simeq & \frac{1}{2} \zeta^{\prime}(0)+a_{0} \frac{T^{3}}{\hbar^{3}} \frac{2 \pi^{2}}{45}+a_{1 / 2} \frac{T^{2}}{\hbar^{2}} \frac{3}{4} \frac{\zeta_{\mathrm{R}}(3)}{\pi^{3 / 2}}+a_{1} \frac{T}{12 \hbar} \\
& +\frac{a_{3 / 2}}{(4 \pi)^{3 / 2}}\left(1-\ln \frac{\hbar}{T}\right)-a_{2} \frac{\hbar}{16 \pi^{2} T}-\frac{a_{5 / 2}}{(4 \pi)^{3 / 2}} \frac{\hbar^{2}}{24 T^{2}} \\
& -\frac{1}{4 \pi^{3 / 2}} \sum_{n \geq 3} a_{n}\left(\frac{\hbar}{2 \pi T}\right)^{2 n-3}(n-2) \Gamma(n-3 / 2) \zeta_{\mathrm{R}}(2 n-3) .
\end{aligned}
$$

In Eq. (2.13) the term proportional to $a_{2}$ contains the logarithm of dimensional quantity: $[\hbar / T]=[\text { time }]^{-1}$. This is the result of the arbitrariness arising in from the ultraviolet divergences in the case of $a_{2} \neq 0$ (see Ref. [21] for a more detailed discussion). Unlike this situation, collecting the logarithm functions in the $a_{3 / 2}$-term and in $\zeta^{\prime}(0)$ in Eq. (2.14) leads to a dimensionless argument of the logarithm in the final expression. 
It is worth noting that the zeta determinant of the operator $-\Delta$ (i. e. $\zeta^{\prime}(0)$ ) does not enter the asymptotic expansion for the internal energy (2.13). Therefore this high temperature expansion is completely defined only by the heat kernel coefficients. In view of this, the first term in the asymptotics of the free energy in Eq. (2.10) is referred to as a pure entropic contribution. Its physical origin is till now not elucidated.

\section{PERFECTLY CONDUCTING PARALLEL PLATES IN VACUUM}

In this section we demonstrate the application of the high temperature expansions (2.10), (2.13), and (2.14) to a simple problem of electromagnetic field confined between two perfectly conducting parallel plates in vacuum. First, we briefly recall how to construct the zeta function in this problem.

As well known, for example, from the theory of waveguides and resonators [22] the vectors of electric and magnetic fields in the problem at hand are expressed in terms of the electric $\left(\boldsymbol{\Pi}^{\prime}\right)$ and magnetic $\left(\boldsymbol{\Pi}^{\prime \prime}\right)$ Hertz vectors, each having only one nonzero component $\Pi_{z}^{\prime}$ and $\Pi_{z}^{\prime \prime}$ satisfying, respectively, Dirichlet and Neumann conditions on the internal surface of the plates. The functions $\Pi_{z}^{\prime}$ and $\Pi_{z}^{\prime \prime}$ obey the equations

$$
\left(\frac{\partial^{2}}{\partial z^{2}}+\nabla^{2}\right) \Pi_{z}^{\prime}=\frac{\omega^{2}}{c^{2}} \Pi_{z}^{\prime}, \quad\left(\frac{\partial^{2}}{\partial z^{2}}+\nabla^{2}\right) \Pi_{z}^{\prime \prime}=\frac{\omega^{2}}{c^{2}} \Pi_{z}^{\prime \prime},
$$

where $\omega$ is the frequency of electromagnetic oscillations, $\nabla^{2}$ stands for the two-dimensional Laplace operator for the variables $(x, y)=\mathbf{x}$. The separation of variables results in the following solution

$$
\begin{aligned}
\Pi_{z}^{\prime}(\mathbf{x}, z) & =\exp (i \mathbf{k x}) \sin \left(\frac{n \pi z}{a}\right), \quad n=1,2, \ldots, \\
\Pi_{z}^{\prime \prime}(\mathbf{x}, z) & =\exp (i \mathbf{k x}) \cos \left(\frac{n \pi z}{a}\right), \quad n=0,1,2, \ldots, \\
\omega_{n}^{2}(\mathbf{k}) & =c^{2}\left[\mathbf{k}^{2}+\left(\frac{n \pi}{a}\right)^{2}\right],
\end{aligned}
$$

where $a$ is the distance between the plates. Hence, the states of electromagnetic field with the energy $\hbar \omega_{n}, \quad n \geq 1$, are doubly degenerate, while the state with the energy $\hbar \omega_{0}=\hbar c k$ is nondegenerate.

With allowance for this the zeta function in the problem under consideration is given by

$$
\zeta(s)=\frac{L_{x} L_{y}}{c^{2 s}} \int \frac{d^{2} \mathbf{k}}{(2 \pi)^{2}}\left\{2 \sum_{n=1}^{\infty}\left[\mathbf{k}^{2}+\left(\frac{n \pi}{a}\right)^{2}\right]^{-s}+\left(\mathbf{k}^{2}+\mu^{2}\right)^{-s}\right\}
$$

where $L_{x}$ and $L_{y}$ are the dimensions of the plates.

For a correct definition of the integral in this formula in the small $\mathbf{k}$ region the photon mass $\mu$ is introduced (infrared regularization). At the final step of calculations one should put $\mu=0$. On integrating in Eq. (3.3) and substituting the sum over $n$ by the Riemann zeta function one arrives at the result 


$$
\zeta(s)=\frac{L_{x} L_{y}}{2 \pi c^{2 s}}\left[\left(\frac{\pi}{a}\right)^{2-2 s} \frac{\zeta_{\mathrm{R}}(2 s-2)}{s-1}+\frac{1}{2} \frac{\mu^{2-2 s}}{s-1}\right] .
$$

The zeta function (3.4) gives the well-known value for the Casimir energy

$$
E_{\mathrm{C}}=\frac{\hbar}{2} \zeta\left(-\frac{1}{2}\right)=-c \hbar \frac{\pi^{2}}{720} \frac{L_{x} L_{y}}{a^{3}}
$$

or for its density

$$
\frac{E_{\mathrm{C}}}{V}=-\frac{c \hbar \pi^{2}}{720 a^{4}}, \quad \text { where } \quad V=a L_{x} L_{y}
$$

In order to construct the high temperature expansions (2.10), (2.13), and (2.14) the heat kernel coefficients for the system under consideration should be obtained by making use of the zeta function (3.4).

The zeta function (3.4) or, in the general case, (2.5) and the corresponding heat kernel (2.8) are related via the Mellin transform

$$
\zeta(s)=\frac{1}{\Gamma(s)} \int_{0}^{\infty} d t t^{s-1} K(t)
$$

This enables one to express the heat kernel coefficients $a_{n}$ in terms of the values of the zeta function at the respective points

$$
\frac{a_{n}}{(4 \pi)^{3 / 2}}=\lim _{s \rightarrow \frac{3}{2}-n}(s+n-3 / 2) \zeta(s) \Gamma(s), \quad n=0,1 / 2, \ldots
$$

Substituting Eq. (3.4) into Eq. (3.8) we obtain for perfectly conducting parallel plates only one nonzero coefficient $a_{0}$

$$
a_{0}=2 \frac{V}{c^{2}}
$$

where $V=L_{x} L_{y} a$ is the volume of the space bounded by the plates.円 This is just an illustration of the well-known fact that for flat manifolds without boundary or with flat boundary all the heat kernel coefficients except for $a_{0}$ vanish [23]. It should be noted here that we are considering only electromagnetic field confined between the plates and do not take into account that field outside the plates.

From Eqs. (2.13) and (3.9) it follows that the density of internal energy has the following high temperature asymptotics

$$
\frac{U(T)}{V} \simeq 4 \frac{\sigma}{c} T^{4}, \quad T \rightarrow \infty
$$

\footnotetext{
${ }^{1}$ For obtaining the vanishing $a_{1 / 2}$ coefficient it is important to take into account the second term in Eq. (3.4) which depends on the photon mass $\mu$.
} 
where $\sigma$ is the Stefan-Boltzmann constant

$$
\sigma=\frac{\pi^{2} k_{\mathrm{B}}^{4}}{60 c^{2} \hbar^{3}} .
$$

Recall that in our formulae we put $k_{\mathrm{B}}=1$, that is, the temperature is measured in energy units. The transition to degrees is performed by the substitution $T \rightarrow k_{\mathrm{B}} T$.

When calculating the high temperature asymptotics of the free energy (2.10) and the entropy (2.14) one needs to derive $\zeta^{\prime}(0)$ for the zeta function (3.4). Keeping in mind that $\zeta_{\mathrm{R}}(-2)=0$ it is convenient to use here the Riemann reflection formula

$$
2^{1-s} \Gamma(s) \zeta_{\mathrm{R}}(s) \cos (\pi s / 2)=\pi^{2} \zeta_{\mathrm{R}}(1-s)
$$

which yields

$$
\zeta_{\mathrm{R}}(2 s-2) \underset{s \rightarrow 0}{\simeq}-s \frac{\zeta_{\mathrm{R}}(3)}{2 \pi^{2}}+\mathcal{O}\left(s^{2}\right) .
$$

From here we deduce

$$
\zeta^{\prime}(0)=\frac{L_{x} L_{y}}{4 \pi a^{2}} \zeta_{\mathrm{R}}(3)=\frac{V}{4 \pi a^{3}} \zeta_{\mathrm{R}}(3) .
$$

Insertion of Eqs. (3.9) and (3.14) into Eq. (2.10) gives the following high temperature behaviour for the density of free energy

$$
\frac{F}{V} \simeq-\frac{T}{8 \pi a^{3}} \zeta_{\mathrm{R}}(3)-\frac{T^{4}}{c^{3} \hbar^{3}} \frac{\pi^{2}}{90} .
$$

As was noted above, we are considering only electromagnetic field between the plates. Therefore when calculating the Casimir forces one should drop the last term in Eq. (3.15) since its contribution is canceled by the pressure of the black body radiation on the outward surfaces of the plates. As a result the high temperature asymptotics of the Casimir force, per unit surface area, attracting two perfectly conducting plates in vacuum is

$$
\mathcal{F} \simeq-\frac{T}{4 \pi a^{3}} \zeta_{\mathrm{R}}(3) .
$$

Usually in the Casimir calculations the contribution of the free black body radiation is subtracted from the very beginning [24].

It is interesting to note that the Casimir force (3.16) and the first term on the right hand side of Eq. (3.15) are pure classical quantities because they do not involve the Planck constant $\hbar$. These classical asymptotics seem to be derivable without appealing to the notion of quantized electromagnetic field. The classical limit of the theory of the Casimir effect is discussed in a recent paper [13].

Employing Eqs. (2.12) and (3.15) one arrives at the high temperature behavior of the entropy density

$$
\frac{S(T)}{V} \simeq \frac{\zeta_{\mathrm{R}}(3)}{8 \pi a^{3}}+\frac{2 T^{3} \pi^{2}}{45 c^{3} \hbar^{3}} .
$$


It is worth noting that the corrections to Eqs. (3.10), (3.15), (3.17) are exponentially small.

The example considered shows that the zeta function of the spatial part of evolution operator really enables one to obtain the high temperature asymptotics of the thermodynamic functions in a straightforward way. In the subsequent sections we shall consider quantum fields defined on manifolds with boundaries possessing spherical or cylindrical symmetries, when the relevant zeta functions cannot be obtained in a closed form. Furthermore in these cases the spectrum of the operator $-\Delta$ is not known explicitly. And nevertheless the method proposed is applicable to these cases also.

\section{THERMODYNAMIC ASYMPTOTICS FOR ELECTROMAGNETIC FIELD WITH BOUNDARY CONDITIONS ON A SPHERE}

In the present section we consider electromagnetic field subjected to three types of boundary conditions on the surface of a sphere: i) an infinitely thin and perfectly conducting spherical shell; ii) the surface of a sphere delimits two material media with the same velocity of light; iii) a dielectric ball placed in unbounded dielectric medium. In order to obtain the heat kernel coefficients determining the high temperature asymptotics (2.10), (2.13), and (2.14) it is convenient to use the explicit representation of the relevant spectral zeta functions in terms of the Riemann zeta function. These formulae were derived in our recent paper [18] by taking into account the first two terms of the uniform asymptotic expansion for the product of the modified Bessel functions $I_{\nu}(\nu z) K_{\nu}(\nu z)$.

\section{A. Perfectly conducting spherical shell}

We take advantage of Eq. (2.26) in Ref. [18 substituting there the variable $s$ by $2 s$ and recovering the explicit dependence on the velocity of light $c$. The latter results in the replacement of the sphere radius by $R / c$ :

$$
\begin{aligned}
\zeta(s) & \simeq \frac{1}{4}\left(\frac{R}{c}\right)^{2 s} s(1+s)(2+s)\left\{\left(2^{1+2 s}-1\right) \zeta_{\mathrm{R}}(1+2 s)-2^{1+2 s}\right. \\
& \left.+q(s)\left[\left(2^{3+2 s}-1\right) \zeta_{\mathrm{R}}(3+2 s)-2^{3+2 s}\right]+\ldots\right\}
\end{aligned}
$$

where

$$
q(s)=\frac{1}{3840}\left(480+1736+2016 s^{2}+568 s^{3}\right)
$$

and $R$ is the radius of a sphere. The terms omitted in Eq. (4.1) are of the form

$$
q_{k}(s)\left[\left(2^{2(k+s)+1}-1\right) \zeta_{\mathrm{R}}(2 k+2 s+1)-2^{2(k+s)+1}\right], \quad k=2,3,4, \ldots,
$$

where $q_{k}(s)$ stand for some polynomials in $s$.

Analysis of Eqs. (4.1) and (4.2) shows that the zeta function (4.1) for a perfectly conducting spherical shell enables one to find the exact values of the first six heat kernel coefficients, namely: 


$$
a_{0}=0, \quad a_{1 / 2}=0, \quad a_{1}=0, \quad a_{3 / 2}=2 \pi^{3 / 2}, \quad a_{2}=0, \quad a_{5 / 2}=\frac{\pi^{3 / 2}}{20} \frac{c^{2}}{R^{2}}
$$

Taking into account the structure of the omitted terms $(4.3)$ it is easy to see that

$$
a_{j}=0, \quad j=3,4,5, \ldots
$$

Having obtained the heat kernel coefficients (4.4) and (4.5) we are in position to construct the high temperature asymptotics of the internal energy of electromagnetic field by making use of Eq. 2.13)

$$
U(T) \simeq \frac{T}{4}-\left(\frac{c \hbar}{R}\right)^{2} \frac{1}{1920 T}+\mathcal{O}\left(T^{-3}\right) .
$$

Applying the technique developed in Ref. 25] more terms to this expansion can be easily added.

In order to write the asymptotic expansions (2.10) and (2.14) the derivative of the zeta function at the point $s=0$ should be calculated. Equation (4.1) gives an approximate value for $\zeta^{\prime}(0)$

$$
\zeta^{\prime}(0)=\frac{\gamma}{2}+\ln 2+\frac{7}{16} \zeta_{\mathrm{R}}(3)-\frac{9}{8}+\frac{1}{2} \ln \frac{R}{c}=0.38265+\frac{1}{2} \ln \frac{R}{c} .
$$

The terms omitted in (4.1) will render precise only the first term in the final form of this expression, while the second term $(1 / 2) \ln (R / c)$ will not change. The exact value of $\zeta^{\prime}(0)$ is calculated in Appendix A

$$
\begin{aligned}
\zeta^{\prime}(0) & =\frac{1}{2}-\frac{\gamma}{2}+\frac{7}{6} \ln 2+6 \zeta_{\mathrm{R}}^{\prime}(-1)+\left(-\frac{5}{8}+\frac{1}{2} \ln \frac{R}{c}+\ln 2+\frac{\gamma}{2}\right) \\
& =0.38429+\frac{1}{2} \ln \frac{R}{c} .
\end{aligned}
$$

It is worth noting that the expression in the round parentheses, being multiplied by $\xi^{2}$, is exactly the value of $\zeta^{\prime}(0)$ for a compact ball with continuous velocity of light on its surface (see Eq. (4.20) in the next subsection). As a result we have the following high temperature asymptotics of the free energy and the entropy in the problem in question

$$
\begin{aligned}
& F(T) \simeq-\frac{T}{4}\left(\ln \frac{R T}{\hbar c}+0.76858\right)-\left(\frac{\hbar c}{R}\right)^{2} \frac{1}{3840 T}+\mathcal{O}\left(T^{-3}\right), \\
& S(T) \simeq 0.44215+\frac{1}{4} \ln \frac{R T}{\hbar c}-\frac{1}{3840}\left(\frac{\hbar c}{R T}\right)^{2}+\mathcal{O}\left(T^{-4}\right) .
\end{aligned}
$$

The expression (4.9) exactly reproduces the asymptotics obtained in Ref. [14] by making use of the multiple scattering technique (see Eq. (8.39) in that paper). We have not calculated the coefficient $a_{7 / 2}$, therefore we do not know the sign of the $T^{-3}$-correction in (4.9). In Ref. [14] it is noted that this term is negative. 
In Eqs. (4.6), (4.9), and (4.10) the large expansion parameter is actually a dimensionless 'temperature' $\tau=R T /(\hbar c)$. Therefore the same formulae describe the behavior of the thermodynamic functions when $R \rightarrow \infty$ and temperature $T$ is fixed.

The high temperature asymptotics of the thermodynamic functions derived by making use of the general expansions (2.10), (2.13), and (2.14) contain the terms independent of the Planck constant $\hbar$ or, in other words, classical contributions (see Eqs. (4.6), (4.9), and (4.10)). This is also true for the high temperature limit of the Casimir force calculated per unit area of a sphere

$$
\mathcal{F}(T) \simeq-\frac{1}{4 \pi R^{2}} \frac{\partial F(T)}{\partial R}=\frac{T}{16 \pi R^{3}}-\left(\frac{\hbar c}{R}\right)^{2} \frac{1}{4 \pi R^{3}} \frac{1}{1920 T}+\mathcal{O}\left(T^{-3}\right) .
$$

The leading classical term in the asymptotics (4.11) describes the Casimir force that seeks to expand the sphere. The quantum correction in this formula stands for the Casimir pressure exerted on the sphere surface.

In Eqs. (4.6), (4.9) and (4.10) the Stefan-Boltzmann terms proportional to $T^{4}$ are absent because the contribution of the Minkowski space was subtracted from the very beginning in our calculations [18]. As a result we obtain the vanishing heat kernel coefficient $a_{0}$ which, in general case, is equal to the volume of the system under study [12]. Therefore our results describe only the deviation from the Stefan-Boltzmann law caused by the perfectly conducting sphere.

The vanishing of the coefficients $a_{1 / 2}$ and $a_{1}$ in the problem at hand can be explained by taking into account the general properties of the heat kernel coefficients [12] and by making use of the results obtained in Ref. [25]. As known [26] the solutions to the Maxwell equations with allowance for a perfectly conducting sphere are expressed in terms of the two scalar functions that satisfy the Laplace equation with the Dirichlet and Robin boundary conditions on internal and external surfaces of the sphere. In view of this one can write

$$
a_{n}=a_{n+}^{\mathrm{D}}+a_{n-}^{\mathrm{D}}+a_{n+}^{\mathrm{R}}+a_{n-}^{\mathrm{R}}, \quad n=1 / 2,1, \ldots,
$$

where subscribe plus (minus) corresponds to internal (external) region and the rest notations are obvious. In Ref. [25] it was found that

$$
\begin{gathered}
a_{1 / 2+}^{\mathrm{D}}=-2 \pi^{3 / 2} R^{2}=a_{1 / 2-}^{\mathrm{D}}, \quad a_{1 / 2+}^{\mathrm{R}}=2 \pi^{3 / 2} R^{2}=a_{1 / 2-}^{\mathrm{R}}, \\
a_{1 \pm}^{\mathrm{D}}= \pm \frac{8 \pi R}{3}, \quad a_{1 \pm}^{\mathrm{R}}=\mp \frac{16 \pi R}{3} .
\end{gathered}
$$

As a result we have

$$
a_{1 / 2}=a_{1}=0 .
$$

Having calculated the corrections to the Stefan-Boltzmann law one should naturally discuss the possibility of their detection. The ratio of the leading term in Eq. (4.6) to the internal energy of black body radiation in unbounded space given by the Stefan-Boltzmann law (3.10) is proportional to $\tau^{-3}$. Already for $\tau \sim 10$ the corrections prove to be of order $10^{-3}$. The same value of $\tau$ can be reached by varying the scale of length $R$ in the problem under consideration or by respective choice of the temperature $T$. Keeping in mind the 


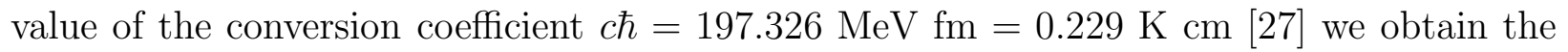
following estimations. For $R \sim 10^{-13} \mathrm{~cm}$ (a typical hadron size) the temperature $T$ should satisfy the inequality $T \gg 200 \mathrm{MeV}$ in order to apply the asymptotics found. For $R \sim 1 \mathrm{~cm}$ we have $T \gg 0.229 \mathrm{~K}$ and for $R \sim 7 \cdot 10^{10} \mathrm{~cm}$ (radius of the Sun) the range of applicability of the asymptotics at hand extends practically to any temperature value $T \gg 10^{-10} \mathrm{~K}$. Here we shall not go into the details of a concrete experimental equipment that enables one to observe the calculated corrections to the Stefan-Boltzmann law confining ourselves to the estimations presented above.

\section{B. Compact ball with equal velocities of light inside and outside}

Let us consider the spherical surface that delimits the media with 'relativistic invariant' characteristics, i.e., the velocity of light is the same inside and outside the sphere [28]. In this problem there naturally arises a dimensionless parameter [29]

$$
\xi^{2}=\left(\frac{\varepsilon_{1}-\varepsilon_{2}}{\varepsilon_{1}+\varepsilon_{2}}\right)^{2}=\left(\frac{\mu_{1}-\mu_{2}}{\mu_{1}+\mu_{2}}\right)^{2},
$$

where $\varepsilon_{1}$ and $\varepsilon_{2}\left(\mu_{1}\right.$ and $\left.\mu_{2}\right)$ are permittivities (permeabilities) inside and outside the sphere. As usual we perform the calculation in the first order of the expansion with respect to $\xi^{2}$.

In order to derive the zeta function for the boundary conditions at hand one should multiply Eq. (4.1) by $\xi^{2}$ and replace there $q(s)$ by the polynomial

$$
p(s)=-\frac{1}{2}\left[1-\frac{9}{2}(3+s)+\frac{5}{2}(3+s)(4+s)-\frac{7}{24}(3+s)(4+s)(5+s)\right] .
$$

The zeta function, obtained in this way, affords the exact heat kernel coefficients up to $a_{3}$

$$
\begin{gathered}
a_{0}=0, \quad a_{1 / 2}=0, \quad a_{1}=0, \quad a_{3 / 2}=2 \pi^{3 / 2} \xi^{2}, \quad a_{2}=0 \\
\frac{a_{5 / 2}}{(4 \pi)^{3 / 2}}=\xi^{2} \frac{c^{2}}{R^{2}} \frac{p(-1)}{8}=0 .
\end{gathered}
$$

With allowance of the structure of the omitted terms in Eq. (4.1) we can again deduce that

$$
a_{j}=0, \quad j=3,4,5, \ldots
$$

Substitution of these coefficients into Eq. (2.13) gives the following high temperature behavior of the internal energy in the problem under consideration

$$
U(T) \simeq \xi^{2} \frac{T}{4}+\mathcal{O}\left(T^{-3}\right)
$$

The value of $\zeta^{\prime}(0)$ is calculated in Appendix A

$$
\zeta^{\prime}(0)=\xi^{2}\left(-\frac{5}{8}+\frac{1}{2} \ln \frac{R}{c}+\ln 2+\frac{\gamma}{2}\right)=\xi^{2}\left(0.35676+\frac{1}{2} \ln \frac{R}{c}\right)
$$

It is this value that is supplied by Eq. (4.1) after changes specified above and with allowance for that $p(-1)=0$. 
By making use of Eqs. (2.10), (4.17), and (4.20) we deduce the high temperature asymptotics for free energy

$$
\begin{aligned}
F(T) & =-\xi^{2} \frac{T}{4}\left(\gamma+\ln 4-\frac{5}{4}\right)+\frac{\xi^{2}}{4} T \ln \frac{\hbar c}{R T}+\mathcal{O}\left(T^{-3}\right) \\
& =-\xi^{2} \frac{T}{4} 0.71352+\frac{\xi^{2}}{4} T \ln \frac{\hbar c}{R T}+\mathcal{O}\left(T^{-3}\right) .
\end{aligned}
$$

The entropy in the present case has the following high temperature behavior

$$
\begin{aligned}
S(T) & =\frac{\xi^{2}}{4}\left(1+\gamma+\ln 4-\frac{5}{4}-\ln \frac{\hbar c}{R T}\right)+\mathcal{O}\left(T^{-4}\right), \\
& =\frac{\xi^{2}}{4}\left(1.71352-\ln \frac{\hbar c}{R T}\right)+\mathcal{O}\left(T^{-4}\right) .
\end{aligned}
$$

The asymptotics (4.19) and (4.21) completely coincide with the analogous formulae obtained in Refs. [30, 31] by the mode summation method combined with the addition theorem for the Bessel functions.

In Ref. 30 the exact expression has also been derived for the internal energy in the problem at hand (see Eq. (3.22) in that paper). This formula gives only exponentially suppressed corrections to the leading term (4.19)

$$
U(T) \simeq \xi^{2} \frac{T}{4}\left[1+2\left(4 t^{2}+4 t+1\right) e^{-4 t}\right]
$$

where $t=2 \pi R T$. We have used here the relation between $\xi^{2}$ and $\Delta n: \xi^{2}=\Delta n^{2} / 4$ (see Eq. (3.12) in Ref. [30]). The asymptotics (4.23) implies in particular that in reality in Eq. (4.19) there are no corrections proportional to the inverse powers of the temperature $T$. From here it follows immediately that all the heat kernel coefficients with integer and half integer numbers equal or greater than 3 should vanish

$$
a_{j}=0, \quad j=3,7 / 2,4,5 / 2,5, \ldots
$$

(compare with Eq. (4.18)). In view of this the sign denoting the omitted terms in Eqs. (4.19), (4.21), and (4.22) should be substituted by $\mathcal{O}\left(e^{-8 \pi R T}\right)$.

\section{Dielectric ball in unbounded dielectric medium}

The zeta function for electromagnetic field in the background of a pure dielectric ball $\left(\mu_{1}=\mu_{2}=1, \quad \varepsilon_{1} \neq \varepsilon_{2}\right)$ has not been obtained in an explicit form. In Ref. [21] the heat kernel coefficients up to $a_{2}$ in this problem were found. Here we use the results of this paper confining ourselves to the $\Delta n^{2}$-approximation, where $\Delta n=n_{1}-n_{2}=n_{1} n_{2}\left(c_{2}-c_{1}\right) / c \simeq$ $\left(c_{2}-c_{1}\right) / c, n_{i}$ and $c_{i}$ are the refractive index and the velocity of light inside $(i=1)$ and outside $(i=2)$ the ball, and $c$ is the velocity of light in the vacuum: $n_{i}=\sqrt{\varepsilon_{i}}, \quad c_{i}=c / n_{i}, \quad i=1,2$. It is assumed that $c_{1}$ and $c_{2}$ differ from $c$ slightly, therefore $c_{2}-c_{1}$ and $\Delta n$ are small quantities. In view of this we have 


$$
\begin{gathered}
a_{0}=\frac{8}{3} \pi R^{3} \frac{c_{2}^{3}-c_{1}^{3}}{c_{1}^{3} c_{2}^{3}} \simeq 8 \pi \frac{R^{3}}{c^{3}}\left(\Delta n+2 \Delta n^{2}\right), \\
a_{1 / 2}=-2 \pi^{3 / 2} R^{2} \frac{\left(c_{1}^{2}-c_{2}^{2}\right)^{2}}{c_{1}^{2} c_{2}^{2}\left(c_{1}^{2}+c_{2}^{2}\right)} \simeq-4 \pi^{3 / 2} \frac{R^{2}}{c^{2}} \Delta n^{2}, \\
a_{1} \simeq 0, \quad a_{3 / 2}=\pi^{3 / 2} \frac{\left(c_{1}^{2}-c_{2}^{2}\right)^{2}}{\left(c_{1}^{2}+c_{2}^{2}\right)^{2}} \simeq \pi^{3 / 2} \Delta n^{2}, \quad a_{2} \simeq 0 .
\end{gathered}
$$

The coefficients $a_{1}$ and $a_{2}$ equal zero only in the $\Delta n^{2}$-approximation considered here. In the general case they contain terms proportional to $\Delta n^{k}$, where $k \geq 3$.

Allowance for one more term in the uniform asymptotic expansion of the modified Bessel functions, as compared with the calculations in Ref. [21], gives the next heat kernel coefficient

$$
\frac{a_{5 / 2}}{(4 \pi)^{3 / 2}}=\frac{25}{2688} \frac{c^{4}}{R^{2}} \Delta n^{4} .
$$

Correcting the mistake made in 32 we state that this coefficient has no contributions proportional to $\Delta n^{2}$, and in the $\Delta n^{2}$-approximation one has to put

$$
a_{5 / 2} \simeq 0 \text {. }
$$

Making use of the technique developed in Ref. [20] one obtains the following expression for the derivative of the zeta function for a pure dielectric ball at the point $s=0$ (see Appendix A)

$$
\zeta^{\prime}(0)=\frac{\Delta n^{2}}{4}\left(-\frac{7}{8}+\ln \frac{R}{c}+\ln 4+\gamma\right)
$$

Before turning to the construction of the high temperature asymptotics in the problem at hand by making use of the general formulae (2.10), (2.13), and (2.14) the following remark should be done. When considering the electromagnetic field in the background of a dielectric body in the formalism of quantum electrodynamics of continuous media, as a matter of fact one is dealing with a system consisting of two objects: electromagnetic field plus a continuous dielectric body. It is important that this body is described (phenomenologically) only by respective permittivity without introducing into the Hamiltonian special additional dynamical variables. As a result the zeta function and the relevant heat kernel coefficients calculated in this formalism also describe both electromagnetic field and dielectric body. When we are interested in the Casimir thermodynamic functions in such problems we have obviously to separate in the general expressions the contributions due to the dielectric body itself [33].

Let us turn to such separation procedure in the high temperature asymptotics for a dielectric ball. Following the reasoning of Refs. 34, 35] we divide the Helmholtz free energy of a material body with volume $V$ and the surface area $S$ into the parts

$$
F=V f+S \sigma+F_{\text {Cas }}
$$

where $f$ is the free energy of a unit volume of a ball, $\sigma$ denotes the surface tension, and $F_{\text {Cas }}$ is refered to as the Casimir free energy of electromagnetic field connected with this 
body and having the temperature $T$. In this way we obtain the following high temperature behavior of the free energy $F(T)$ in the problem at hand

$$
F(T) \simeq a_{0} \frac{T^{4}}{\hbar^{3}} \frac{\pi^{2}}{90}-a_{1 / 2} \frac{T^{3}}{4 \pi^{3 / 2} \hbar^{2}} \zeta_{\mathrm{R}}(3)+F_{\mathrm{Cas}}(T),
$$

where $a_{0}$ and $a_{1 / 2}$ are defined in Eq. (4.25) and

$$
F_{\text {Cas }}(T) \simeq-\frac{\Delta n^{2}}{8} T\left(\ln \frac{4 T R}{\hbar c}+\gamma-\frac{7}{8}\right)+\mathcal{O}\left(T^{-2}\right)
$$

The high temperature asymptotics for the Casimir internal energy and for the Casimir entropy can be derived by making use of the respective thermodynamical relations (2.11), (2.12)

$$
\begin{aligned}
U_{\mathrm{Cas}}(T) & \simeq \frac{\Delta n^{2}}{8} T+\mathcal{O}\left(T^{-2}\right), \\
S_{\mathrm{Cas}}(T) & \simeq \frac{\Delta n^{2}}{8}\left(\frac{1}{8}+\gamma+\ln \frac{4 R T}{\hbar c}\right)+\mathcal{O}\left(T^{-3}\right) .
\end{aligned}
$$

It is worth comparing these results with analogous asymptotics obtained by different methods. In Ref. [30] at the beginning of calculations the first term of expansion of internal energy (4.32) was derived. The subsequent integration of the thermodynamic relation (2.11) gave the correct coefficient of the logarithmic term in the asymptotics of free energy (4.31). In a very recent paper [35] Barton managed to deduce the asymptotics (4.31) - (4.33). One should keep in mind that our parameter $\Delta n$ corresponds to $2 \pi \alpha n$ in the notations of Ref. [35].

The asymptotics (4.31)-(4.33) contain the $R$-independent terms. As far as we know the physical meaning of such terms remains unclear.

Preliminary analysis of a complete expression for the internal energy of a dielectric ball (see Eqs. (3.20) and (3.31) in Ref. [30]) shows that probably there are only exponentially suppressed corrections to the leading term (4.32). In that case in addition to Eq. (4.27) all the heat kernel coefficients with number greater than 3 should vanish in the $\Delta n^{2}$-approximation.

\section{THERMODYNAMIC ASYMPTOTICS FOR ELECTROMAGNETIC FIELD WITH BOUNDARY CONDITIONS ON AN INFINITE CYLINDER}

The calculation of the vacuum energy of electromagnetic field with boundary conditions defined on a cylinder, to say nothing of the temperature corrections, turned out to be technically a more involved problem than the analogous one for a sphere. Therefore the Casimir problem for a cylinder has been considered only in a few papers [14, 18, 19, 36] 39]. We again examine three cases: i) perfectly conducting cylindrical shell; ii) solid cylinder with $c_{1}=c_{2}$; iii) dielectric cylinder when $c_{1} \neq c_{2}$. Here we shall use the results of our previous papers [18, 19]. 


\section{A. Perfectly conducting cylindrical shell}

In Ref. [18] the first two terms in the uniform asymptotic expansion of the product of the modified Bessel functions $I_{n}(n x) K_{n}(n x)$ were taken into account. As a result the spectral zeta function in the problem under consideration was represented as an expansion in terms of the Riemann zeta functions $\zeta_{\mathrm{R}}(2(k+s)+1), \quad k=0,1,2, \ldots$. With allowance for the first two terms in this expansion the zeta function is given by

$$
\zeta(s)=Z_{1}(s)+Z_{2}(s)+Z_{3}(s) .
$$

Here the function $Z_{1}(s)$ stands for the contribution of zero orbital momentum with proper subtraction

$$
\begin{gathered}
Z_{1}(s)=\frac{(2 s-1) R^{2 s-1}}{2 \sqrt{\pi} c^{2 s} \Gamma(s) \Gamma(3 / 2-s)} \int_{0}^{\infty} d y y^{-2 s}\left\{\ln \left[1-\mu_{0}^{2}(y)\right]+\frac{1}{4} y^{2} t^{6}(y)\right\} \\
\mu_{n}(y)=y\left(I_{n}(y) K_{n}(y)\right)^{\prime}, \quad t(y)=\frac{1}{\sqrt{1+y^{2}}} .
\end{gathered}
$$

The function $Z_{2}(s)$ is generated by the first term of the uniform asymptotic expansion

$$
Z_{2}(s)=\frac{R^{2 s-1}}{64 \sqrt{\pi} c^{2 s}}(1-2 s)(3-2 s)\left[2 \zeta_{\mathrm{R}}(2 s+1)+1\right] \frac{\Gamma(1 / 2+s)}{\Gamma(s)} .
$$

The function $Z_{3}$ corresponds to the second term of the uniform asymptotic expansion

$$
Z_{3}(s)=\frac{R^{2 s-1}}{61440 \sqrt{\pi}}(1-2 s)(3-2 s)\left(784 s^{2}-104 s-235\right) \frac{\Gamma(3 / 2+s)}{\Gamma(s)} \zeta_{\mathrm{R}}(2 s+3) .
$$

The function $Z_{1}(s)$ is defined in the strip $-3 / 2<\Re s<1 / 2$, while the functions $Z_{2}(s)$ and $Z_{3}(s)$ are analytic functions in the whole complex plane $s$ except for the points, where $\Gamma(s)$ and $\zeta_{\mathrm{R}}(s)$ have simple poles. In order to find the heat kernel coefficients $a_{0}, a_{1 / 2}$, and

$a_{1}$ trough the relation (3.8) one needs the zeta function defined in the region $1 / 2+\varepsilon \leq$ $\Re s \leq 3 / 2+\varepsilon$ with $\varepsilon$ being a positive infinitesimal. However in this region Eq. (5.2) is not applicable directly due to the bad behaviour of the integral at the upper limit. In the most simple way we can overcome this difficulty as in the case of perfectly conducting plates by introducing the photon mass $\mu$ at the very beginning of the calculation and making then the analytic continuation of the zeta function to the points $s=1 / 2,1,3 / 2$. Upon taking the residua at these points one should put $\mu=0$.

With regard to all this and using the relation (3.8) we find the heat kernel coefficients

$$
a_{0}=0, \quad a_{1 / 2}=0, \quad a_{1}=0, \quad a_{2}=0 .
$$

The vanishing heat kernel coefficient $a_{2}$ implies that the zeta regularization gives a finite value for the vacuum energy in the problem at hand [18,37. The coefficient $a_{3 / 2}$ is determined by the function $Z_{2}(s)$ only (see Eq. (5.3) )

$$
\frac{a_{3 / 2}}{(4 \pi)^{3 / 2}}=\frac{3}{64 R}
$$


The coefficient $a_{5 / 2}$ is defined by the function $Z_{3}(s)$ given in Eq. (5.4)

$$
\frac{a_{5 / 2}}{(4 \pi)^{3 / 2}}=\frac{153}{8192} \frac{c^{2}}{R^{3}} .
$$

The calculation of the next heat kernel coefficients $a_{3}, a_{7 / 2}, \ldots$ would demand a knowledge of the additional terms in the expansion of the spectral zeta function in the problem under consideration in terms of the Riemann zeta function. These terms are proportional to $\zeta_{\mathrm{R}}(2 k+2 s+1)$ with $k=2,3, \ldots$, and may be obtained employing the technique developed in Ref. [18]. Analyzing the position of poles for these Riemann zeta functions it is easy to show that, as well as in the spherical case, we have

$$
a_{j}=0, \quad j=3,4,5 \ldots
$$

The zeta determinant entering the high temperature asymptotics of free energy (2.10) and entropy (2.14) is calculated in Appendix B

$$
\zeta^{\prime}(0)=\frac{0.45847}{R}+\frac{3}{32 R} \ln \frac{R}{2 c} .
$$

Now we are able to construct the high temperature expansions of the thermodynamic functions in the problem under consideration. For the free energy we have

$$
F(T) \simeq-0.22924 \frac{T}{R}-\frac{3 T}{64 R} \ln \frac{R T}{2 \hbar c}-\frac{51}{65536} \frac{\hbar^{2} c^{2}}{R^{3} T}+\mathcal{O}\left(T^{-3}\right) .
$$

When comparing Eq. (5.9) with results of other authors one should remember that all the thermodynamic quantities that we obtained in this section are related to a cylinder of unit length. The high temperature asymptotics of the electromagnetic free energy in presence of perfectly conducting cylindrical shell was investigated in Ref. [14]. To make the comparison convenient we rewrite their result as follows

$$
F(T) \simeq-0.10362 \frac{T}{R}-\frac{3 T}{64 R} \ln \frac{R T}{2 \hbar c} .
$$

The discrepancy between the terms linear in $T$ in Eqs. (5.9) and (5.10) is due to the double scattering approximation used in Ref. [14] (see also the next subsection). Our approach provides an opportunity to calculate the exact value of this term (see Eq. (5.9)).

And finally, making use of the general formulae (2.13) and (2.14) we derive

$$
\begin{aligned}
U(T) & \simeq \frac{3 T}{64 R}-\frac{153}{98304} \frac{c^{2} \hbar^{2}}{R^{3} T}+\mathcal{O}\left(T^{-3}\right), \\
S(T) & \simeq \frac{0.27612}{R}+\frac{3}{64 R} \ln \frac{R T}{2 \hbar c}-\frac{153}{196608} \frac{c^{2} \hbar^{2}}{R^{3} T^{2}}+\mathcal{O}\left(T^{-4}\right) .
\end{aligned}
$$




\section{B. Compact cylinder with $c_{1}=c_{2}$ and with $c_{1} \neq c_{2}$}

Here we consider the boundary conditions for electromagnetic field of two types: i) a compact infinite cylinder with uniform velocity of light on its lateral surface, ii) a pure dielectric cylinder with $c_{1} \neq c_{2}$. The explicit expressions for the heat kernel coefficients up to $a_{2}$ we take from Ref. [19], where a compact cylinder with unequal velocities of light inside

and outside was considered. When $c_{1}=c_{2}$ the final expressions for these coefficients are drastically simplified

$$
a_{0}=0, \quad a_{1 / 2}=0, \quad a_{1}=0, \quad \frac{a_{3 / 2}}{(4 \pi)^{3 / 2}}=\frac{3 \xi^{2}}{64 R}, \quad a_{2}=0 .
$$

The zeta function obtained for given boundary conditions in Ref. 18 gives

$$
\frac{a_{5 / 2}}{(4 \pi)^{3 / 2}}=\xi^{2} \frac{c^{2}}{R^{3}} \frac{45}{8192}, \quad a_{j}=0, \quad j=3,4,5, \ldots
$$

The heat kernel coefficients (5.13) and (5.14) lead to the following high temperature behavior of the internal energy in the problem at hand

$$
U(T)=\frac{3 \xi^{2} T}{64 R}\left(1-\frac{5}{512} \frac{c^{2} \hbar^{2}}{R^{2} T^{2}}\right)+\mathcal{O}\left(T^{-3}\right) .
$$

The corresponding zeta determinant is calculated in Appendix B

$$
\zeta^{\prime}(0)=\frac{\xi^{2}}{R}\left(0.20699+\frac{3}{32} \ln \frac{R}{2 c}\right)
$$

Now we can write the high temperature asymptotics for free energy

$$
F(T)=-\xi^{2} \frac{T}{R}\left[0.10350+\frac{3}{64} \ln \frac{T R}{2 \hbar c}+\frac{15}{65536} \frac{c^{2} \hbar^{2}}{R^{2} T^{2}}\right]+\mathcal{O}\left(T^{-3}\right)
$$

and for entropy

$$
S(T)=\frac{\xi^{2}}{R}\left[0.10350+\frac{3}{64}\left(1+\ln \frac{R T}{2 \hbar c}\right)-\frac{15}{65536} \frac{c^{2} \hbar^{2}}{T^{2} R^{2}}\right]+\mathcal{O}\left(T^{-4}\right) .
$$

Putting in these equations $\xi^{2}=1$ we arrive at the double scattering approximation for a perfectly conducting cylindrical shell (see Eq. (5.10)). A slight distinction between the linear in $T$ terms in Eq. (5.10) and Eq. (5.17) is due to a finite error inherent in the numerical methods employed in both the approaches.

In the case of a pure dielectric cylinder $\left(\mu_{1}=\mu_{2}=1, \varepsilon_{1} \neq \varepsilon_{2}\right)$ the first four heat kernel coefficients are different from zero even in the dilute approximation [19] (small difference between the velocities of light inside and outside the cylinder) 


$$
\begin{array}{r}
a_{0}=-\frac{6 \pi R^{2}}{c_{2}^{4}}\left(c_{1}-c_{2}\right)+\frac{12 \pi R^{2}}{c_{2}^{5}}\left(c_{1}-c_{2}\right)^{2}, \quad a_{1 / 2}=-\frac{2 \pi^{3 / 2} R}{c_{2}^{4}}\left(c_{1}-c_{2}\right)^{2}, \\
a_{1}=\frac{8 \pi}{c_{2}^{2}}\left(c_{1}-c_{2}\right)-\frac{14 \pi}{3 c_{2}^{3}}\left(c_{1}-c_{2}\right)^{2}, \quad a_{3 / 2}=\frac{3 \pi^{3 / 2}}{16 R c_{2}^{2}}\left(c_{1}-c_{2}\right)^{2}, \\
a_{2}=0, \quad \frac{a_{5 / 2}}{(4 \pi)^{3 / 2}}=\frac{857}{61440} \frac{\left(c_{1}-c_{2}\right)^{2}}{R^{3}} .
\end{array}
$$

It should be noted that the coefficient $a_{2}$ vanishes only in the $\left(c_{1}-c_{2}\right)^{2}$-approximation. As a matter of fact $a_{2}$ contains nonvanishing $\left(c_{1}-c_{2}\right)^{3}$-terms and those of higher order [19]. Therefore the zeta regularization provides a finite answer for the vacuum energy of a pure dielectric cylinder only in the $\left(c_{1}-c_{2}\right)^{2}$-approximation even at zero temperature.

The contribution to the asymptotic expansions of the first three heat kernel coefficients should be involved into the relevant phenomenological parameters in the general expression of the classical energy of a dielectric cylinder (in the same way as it has been done for a pure dielectric ball). By making use of the coefficients $a_{3 / 2}$ and $a_{5 / 2}$ we get the high temperature asymptotics of the internal energy in the problem at hand

$$
U(T)=\Delta n^{2} \frac{3}{128} \frac{T}{R}\left(1-\frac{857}{17280} \frac{c^{2} \hbar^{2}}{T^{2} R^{2}}\right)+\mathcal{O}\left(T^{-2}\right) .
$$

where $\Delta n=n_{1}-n_{2} \simeq\left(c_{2}-c_{1}\right) / c$.

In view of a considerable technical difficulties we shall not calculate the zeta function determinant for a pure dielectric cylinder. We recover the respective asymptotics of free energy by integrating the thermodynamic relation (2.11) and of entropy by using the relation (2.12). Pursuing this way we introduce a new constant of integration $\alpha$ that remaines undetermined in our consideration

$$
\begin{aligned}
& F(T)=-\Delta n^{2} \frac{3}{128} \frac{T}{R}\left(\alpha+\ln \frac{R T}{\hbar c}+\frac{857}{34560} \frac{c^{2} \hbar^{2}}{T^{2} R^{2}}\right)+\mathcal{O}\left(T^{-2}\right) \\
& S(T)=\Delta n^{2} \frac{3}{128}\left(1+\alpha+\ln \frac{R T}{\hbar c}-\frac{857}{34560} \frac{c^{2} \hbar^{2}}{T^{2} R^{2}}\right)+\mathcal{O}\left(T^{-3}\right)
\end{aligned}
$$

\section{CONCLUSIONS}

In this paper we have demonstrated efficiency and universality of the high temperature expansions in terms of the heat kernel coefficients for the Casimir problems with spherical and cylindrical symmetries. All the known results in this field are reproduced in a uniform approach and in addition a few new asymptotics are derived (for a compact ball with $c_{1}=c_{2}$ and for a pure dielectric infinite cylinder).

As the next step in the development of this approach one can try to retain the terms exponentially decreasing when $T \rightarrow \infty$. These corrections are well known, for example, for thermodynamic functions of electromagnetic field in the presence of perfectly conducting 
parallel plates [24,40] (see also Eq. (4.23)). In order to reveal such terms, first of all the exponentially decreasing corrections should be retained in the asymptotic expansion (2.8) for the heat kernel.

It is worth noting that in the framework of the method employed the high temperature asymptotics can also be constructed in the problems when the zeta regularization does not provide a finite value of the vacuum energy at zero temperature, i.e. when the heat kernel coefficient $a_{2}$ does not vanish.

In Ref. [13] it was argued that in the high temperature limit the behavior of the Casimir thermodynamic quantities should be the following. In the case of disjoint boundary pieces

the free energy tends to minus infinity, the entropy approaches a constant, and the internal energy vanishes. Contributions to the Casimir thermodynamic quantities due to each individual connected component of the boundary exhibits logarithmic deviations in temperature from the behavior just described. In our consideration we were obviously dealing with an individual connected component of the boundary (a sphere or cylinder). Our results corroborate the relevant conclusions of Ref. [13] concerning the free energy and entropy. However the internal energy in our calculations tends to infinity like $T$ instead to vanish, this increase being caused by the respective logarithmic terms in the high temperature asymptotics of free energy.

\section{ACKNOWLEDGMENTS}

V.V.N. thanks Professor Barton for providing his paper [35] prior publication and for very fruitful communications. The work has been supported by the Heisenberg-Landau Program and by the Russian Foundation for Basic Research (Grant No. 00-01-00300). V.V.N. acknowledges the partial financial support of the International Science and Technology Center (Project No. 840).

\section{APPENDIX A: ZETA FUNCTION DETERMINANTS FOR ELECTROMAGNETIC FIELD SUBJECTED TO SPHERICALLY SYMMETRIC BOUNDARY CONDITIONS}

\section{A perfectly conducting sphere}

First we calculate $\zeta^{\prime}(0)$ (zeta determinant) for electromagnetic field in the background of a perfectly conducting sphere. We proceed from the following representation for this zeta function 18

$$
\zeta(s)=\left(\frac{R}{c}\right)^{2 s} \frac{\sin (\pi s)}{\pi} \sum_{l=1}^{\infty}(2 l+1) \int_{0}^{\infty} d y y^{-2 s} \frac{d}{d y} \ln \left[1-\sigma_{l}^{2}(y)\right],
$$

where

$$
\sigma_{l}(y)=\frac{d}{d y}\left[y I_{\nu}(y) K_{\nu}(y)\right], \quad \nu=l+1 / 2
$$


The analytic continuation of Eq. (A1) to the region $\Im s<0$ is performed by adding and subtracting from the integrand its uniform asymptotics at large $\nu$

$$
\sigma_{l}^{2}(\nu z) \simeq \frac{t^{6}(z)}{4 \nu^{2}}, \quad t(z)=\frac{1}{\sqrt{1+z^{2}}} .
$$

As a result we obtain

$$
\zeta(s)=Z(s)+\zeta_{\mathrm{as}}(s)
$$

where

$$
\begin{aligned}
Z(s) & =\left(\frac{R}{c}\right)^{2 s} \frac{\sin (\pi s)}{2 \pi} \sum_{l=1}^{\infty} \nu^{1-2 s} \int_{0}^{\infty} \frac{d z}{z^{2 s}} \frac{d}{d z}\left\{\ln \left[1-\sigma_{l}^{2}(\nu z)\right]+\frac{1}{4 \nu^{2}} \frac{1}{\left(1+z^{2}\right)^{3}}\right\}, \\
\zeta_{\text {as }}(s) & =\left(\frac{R}{c}\right)^{2 s} \frac{3 \sin (\pi s)}{4 \pi} \sum_{l=1}^{\infty} \nu^{-1-2 s} \int_{0}^{\infty} d z z^{1-2 s} t^{8}(z) \\
& =\frac{1}{4}\left(\frac{R}{c}\right)^{2 s} s(1+s)(2+s)\left[\left(2^{1+2 s}-1\right) \zeta_{\mathrm{R}}(1+2 s)-2^{1+2 s}\right] .
\end{aligned}
$$

When calculating $\zeta^{\prime}(0)$ one can put in Eq. (A5) $s=0$ everywhere except for $\sin (\pi s)$, the latter function being substituted simply by $\pi s$. In view of this the integral in Eq. (A5) is evaluated easy if one takes into account the limits

$$
\lim _{z \rightarrow 0} \sigma_{l}^{2}(\nu z)=\left(\frac{\Gamma(\nu)}{2 \Gamma(\nu+1)}\right)^{2}=\frac{1}{4 \nu^{2}}, \quad \lim _{z \rightarrow 0} \frac{t^{6}(z)}{4 \nu^{2}}=\frac{1}{4 \nu^{2}}
$$

and the asymptotics (A3) at large $z$. As a result we obtain

$$
Z^{\prime}(0)=-2 \sum_{l=1}^{\infty} \nu\left[\ln \left(1-\frac{1}{4 \nu^{2}}\right)+\frac{1}{4 \nu^{2}}\right] .
$$

Differentiation of Eq. (A6) with respect to $s$ at the point $s=0$ gives

$$
\zeta_{\text {as }}^{\prime}(0)=-\frac{5}{8}+\frac{1}{2} \ln R+\ln 2+\frac{\gamma}{2} .
$$

In order to calculate the sum over $l$ in Eq. (A8) we consider an auxiliary sum

$$
S(a)=-\sum_{l=1}^{\infty} 2 \nu\left[\ln \left(1-\frac{a^{2}}{4 \nu^{2}}\right)+\frac{a^{2}}{4 \nu^{2}}\right], \quad S(0)=0, \quad S(1)=Z^{\prime}(0),
$$

where $a$ is a parameter. Derivative of this sum with respect to $a$ can be rewritten in the form

$$
S^{\prime}(a)=-\frac{a}{2} \sum_{l=1}^{\infty}\left[\frac{1}{l+1 / 2}-\frac{1}{l+(1+a) / 2}+\frac{1}{l+1 / 2}-\frac{1}{l+(1-a) / 2}\right]
$$

The summation in Eq. (A11) can be done by making use of the following relations [41] 


$$
\begin{aligned}
& \sum_{k=1}^{\infty}\left(\frac{1}{y+k}-\frac{1}{x+k}\right)=\frac{1}{x}-\frac{1}{y}+\psi(x)-\psi(y), \\
& \psi(x+1)=\psi(x)+\frac{1}{x}, \quad \psi\left(\frac{1}{2}\right)=-\gamma-2 \ln 2,
\end{aligned}
$$

where $\psi(x)$ is the digamma function (the Euler $\psi$ function): $\psi(x)=(d / d x) \ln \Gamma(x)$. This gives

$$
S^{\prime}(a)=a(2-\gamma-2 \ln 2)-\frac{a}{2}\left[\psi\left(\frac{3}{2}+\frac{a}{2}\right)+\psi\left(\frac{3}{2}-\frac{a}{2}\right)\right] .
$$

Now we integrate the both sides of Eq. (A13) over $a$ from 0 to 1 by making use of 'Maple'

$$
S(1)=Z^{\prime}(0)=\frac{1}{2}-\frac{\gamma}{2}+\frac{7}{6} \ln 2+6 \zeta_{\mathrm{R}}^{\prime}(-1) .
$$

From Eqs. (A4), (A9), and (A14) it follows that

$$
\begin{aligned}
\zeta^{\prime}(0) & =\frac{1}{2}-\frac{\gamma}{2}+\frac{7}{6} \ln 2+6 \zeta_{\mathrm{R}}^{\prime}(-1)+\left(-\frac{5}{8}+\frac{1}{2} \ln \frac{R}{c}+\ln 2+\frac{\gamma}{2}\right) \\
& =-\frac{1}{8}+\frac{13}{6} \ln 2+6 \zeta_{\mathrm{R}}^{\prime}(-1)+\frac{1}{2} \ln \frac{R}{c} \\
& =0.38429+\frac{1}{2} \ln \frac{R}{c} .
\end{aligned}
$$

\section{A material ball with $c_{1}=c_{2}$}

The same technique can be used for calculating the zeta function determinant in the case of equal velocities of light inside and outside the material ball (see Section IV.B). The complete zeta function in this problem has the form [18]

$$
\zeta(s)=\left(\frac{R}{c}\right)^{2 s} \frac{\sin (\pi s)}{\pi} \sum_{l=1}^{\infty}(2 l+1) \int_{0}^{\infty} d y y^{-2 s} \frac{d}{d y} \ln \left[1-\xi^{2} \sigma_{l}^{2}(y)\right]
$$

where $\sigma_{l}(y)$ is defined in Eq. (A2) and the parameter $\xi^{2}$ was introduced in Eq. (4.15). Adding and subtracting under the integral sign in Eq. (A16) the uniform asymptotics of the integrand at large $\nu$ we get

$$
\begin{aligned}
\zeta(s)= & \left(\frac{R}{c}\right)^{2 s} \frac{\sin (\pi s)}{2 \pi} \sum_{l=1}^{\infty} \nu^{1-2 s} \int_{0}^{\infty} \frac{d z}{z^{2 s}} \frac{d}{d z}\left\{\ln \left[1-\xi^{2} \sigma_{l}^{2}(\nu z)\right]+\frac{\xi^{2}}{4 \nu^{2}} \frac{1}{\left(1+z^{2}\right)^{3}}\right\} \\
& +\xi^{2} \zeta_{\text {as }}(s),
\end{aligned}
$$

where the function $\zeta_{\text {as }}(s)$ was introduced in Eq. A6). Proceeding in the same way as in the previous subsection we obtain for the derivative of the function $\zeta(s)$ at the point $s=0$

$$
\zeta^{\prime}(0)=S\left(\xi^{2}\right)+\xi^{2} \zeta_{\text {as }}^{\prime}(0)
$$


where the function $S\left(\xi^{2}\right)$ is defined in Eq. (A10). For small values of the argument $\xi^{2}$ we deduce from Eq. (A10)

$$
S\left(\xi^{2}\right)=\frac{\xi^{4}}{16} \sum_{l=1}^{\infty} \frac{1}{\nu^{3}}=\frac{\xi^{4}}{16}\left[7 \zeta_{\mathrm{R}}(3)-8\right]+\mathcal{O}\left(\xi^{6}\right) .
$$

Therefore restricting ourselves to the first order of $\xi^{2}$ we arrive at the final result

$$
\zeta^{\prime}(0)=\xi^{2} \zeta_{\text {as }}^{\prime}(0)=\xi^{2}\left(-\frac{5}{8}+\frac{1}{2} \ln \frac{R}{c}+\ln 2+\frac{\gamma}{2}\right) .
$$

\section{A pure dielectric ball}

A material ball with arbitrary velocities of light inside and outside treated in Section IV.C proves to be a more complicated problem. In notations of Ref. [21] the relevant zeta function takes the form $\zeta(s)=\zeta_{-1}(s)+\zeta_{1}(s)$, where

$$
\zeta_{\rho}(s)=-\frac{2 R^{2 s}}{\Gamma(s+1) \Gamma(-s)} \sum_{l=1}^{\infty} \nu^{1-2 s} \int_{0}^{\infty} d k k^{-2 s} \frac{d}{d k} \ln \Delta_{\rho, l}(\nu k), \quad \nu=l+\frac{1}{2}, \quad \rho= \pm 1
$$

with

$$
\begin{gathered}
\Delta_{\rho, l}(\nu k)=\frac{2 e^{-\left(k_{1}-k_{2}\right) \nu}}{\left(\chi^{\rho}+1\right)}\left[\chi^{\rho} s_{l}^{\prime}\left(\nu k_{1}\right) e_{l}\left(\nu k_{2}\right)-s_{l}\left(\nu k_{1}\right) e_{l}^{\prime}\left(\nu k_{2}\right)\right], \quad k_{1}=k / c_{1}, \quad k_{2}=k / c_{2}, \\
s_{l}(y)=\sqrt{\frac{\pi y}{2}} I_{\nu}(y), \quad e_{l}(y)=\sqrt{\frac{2 y}{\pi}} K_{\nu}(y) .
\end{gathered}
$$

The parameter $\chi=\sqrt{\left(\varepsilon_{1} \mu_{2}\right) /\left(\varepsilon_{2} \mu_{1}\right)}$ corresponds to $\xi$ in Ref. [21].

The analytic continuation of the zeta function at hand to the region $\Re s>0$ is performed by adding and subtracting in Eq. (A22) several terms of its asymptotic expansion

$$
\Delta_{\rho, l}(\nu k) \sim \sum_{n=-1,0,1}^{\infty} \frac{D_{n, \rho}}{\nu^{n}}
$$

where

$$
\begin{aligned}
D_{-1} & =\eta\left(k_{1}\right)-\eta\left(k_{2}\right)-\left(k_{1}-k_{2}\right), \quad \eta(z)=\sqrt{1+z^{2}}+\ln \frac{z}{1+\sqrt{1+z^{2}}}, \\
D_{0} & =\ln \left\{\frac{\chi^{\rho} c_{1} t_{2}+c_{2} t_{1}}{\sqrt{c_{1} c_{2} t_{1} t_{2}}\left(\chi^{\rho}+1\right)}\right\}, \quad t_{i}=\frac{1}{\sqrt{1+k_{i}^{2}}}, \quad i=1,2 .
\end{aligned}
$$

For our purpose it is sufficient to consider four leading terms of the asymptotic expansion (A23), $n=-1,0,1,2$. The functions $D_{1}$ and $D_{2}$ are given in Ref. 21]. Proceeding in this way we represent the zeta function (A21) as follows 


$$
\begin{aligned}
& \zeta_{\rho}(s)=-\frac{2 R^{2 s}}{\Gamma(s+1) \Gamma(-s)} \sum_{l=1}^{\infty} \nu^{-2 s+1} \int_{0}^{\infty} d k k^{-2 s} \frac{d}{d k}\left(\ln \Delta_{\rho, l}-\nu D_{-1}-D_{0}-\frac{D_{1}}{\nu}-\frac{D_{2}}{\nu^{2}}\right) \\
& -\frac{2 R^{2 s}}{\Gamma(s+1) \Gamma(-s)}\left[\zeta_{\mathrm{H}}\left(2 s-2, \frac{3}{2}\right) \int_{0}^{\infty} d k k^{-2 s} \frac{d D_{-1}}{d k}+\zeta_{\mathrm{H}}\left(2 s-1, \frac{3}{2}\right) \int_{0}^{\infty} d k k^{-2 s} \frac{d D_{0}}{d k}\right. \\
& \left.+\zeta_{\mathrm{H}}\left(2 s, \frac{3}{2}\right) \int_{0}^{\infty} d k k^{-2 s} \frac{d D_{1}}{d k}+\zeta_{\mathrm{H}}\left(2 s+1, \frac{3}{2}\right) \int_{0}^{\infty} d k k^{-2 s} \frac{d D_{2}}{d k}\right],
\end{aligned}
$$

where $\zeta_{\mathrm{H}}$ is the Hurwitz zeta function. Taking the derivative of the zeta function (A25) at the point $s=0$ with allowance for the behavior of $D_{i}(k)$ at $k=0$ and $k=\infty$ we obtain

$$
\begin{aligned}
\zeta_{\rho}^{\prime}(0)= & -2 \sum_{l=1}^{\infty}\left(l+\frac{1}{2}\right)\left[\ln \left(1+\frac{1}{2 \nu} \frac{\chi^{\rho} c_{1}-c_{2}}{\chi^{\rho} c_{1}+c_{2}}\right)-\frac{1}{2 \nu} \frac{\chi^{\rho} c_{1}-c_{2}}{\chi^{\rho} c_{1}+c_{2}}+\frac{1}{8 \nu^{2}}\left(\frac{\chi^{\rho} c_{1}-c_{2}}{\chi^{\rho} c_{1}+c_{2}}\right)^{2}\right] \\
& +2\left\{\frac{1}{4} \ln \frac{c_{2}}{c_{1}}+\frac{11}{24} \ln \left[\frac{\chi^{\rho} c_{1}+c_{2}}{\sqrt{c_{1} c_{2}}\left(\chi^{\rho}+1\right)}\right]+\frac{1}{2} \frac{\chi^{\rho} c_{1}-c_{2}}{\chi^{\rho} c_{1}+c_{2}}\right. \\
& \left.-\frac{1}{8}(2-\ln R-\gamma-2 \ln 2)\left(\frac{\chi^{\rho} c_{1}-c_{2}}{\chi^{\rho} c_{1}+c_{2}}\right)^{2}-\int_{0}^{\infty} d k \ln k \frac{d}{d k} D_{2}\right\} .
\end{aligned}
$$

In order to calculate the sum over $l$ in Eq. (A26) we consider an auxiliary sum

$$
S_{1}(b)=\sum_{l=1}^{\infty} 2 \nu\left[\ln \left(1+\frac{b}{2 \nu}\right)-\frac{b}{2 \nu}+\frac{b^{2}}{8 \nu^{2}}\right], \quad S_{1}(0)=0,
$$

with $b$ being a parameter. The derivative of this sum can be cast to the form

$$
S^{\prime}(b)=\frac{b}{2} \sum_{l=1}^{\infty}\left[\frac{1}{l+1 / 2}-\frac{1}{l+(b+1) / 2}\right] .
$$

Taking into account Eqs. (A12) we obtain

$$
S^{\prime}(b)=\frac{b}{2}(-2+\gamma+2 \ln 2)+\frac{b}{2} \psi\left(\frac{b}{2}+\frac{3}{2}\right) .
$$

The integration of Eq. (A29) over $b$ from 0 to $b=\left(\chi^{\rho} c_{1}-c_{2}\right) /\left(\chi^{\rho} c_{1}+c_{2}\right)$ gives the sum entering Eq. A26

$$
\begin{aligned}
& S_{1}(b)=\frac{b^{2}}{4}(-2+\gamma+2 \ln 2)+b \zeta_{\mathrm{H}}^{\prime}\left(0, \frac{3}{2}+\frac{b}{2}\right) \\
& -2\left[\zeta_{\mathrm{H}}\left(-1, \frac{3}{2}+\frac{b}{2}\right)+\zeta_{\mathrm{H}}^{\prime}\left(-1, \frac{3}{2}+\frac{b}{2}\right)\right]+2\left[\zeta_{\mathrm{H}}\left(-1, \frac{3}{2}\right)+\zeta_{\mathrm{H}}^{\prime}\left(-1, \frac{3}{2}\right)\right] .
\end{aligned}
$$

Substitution of Eq. (A30) into Eq. (A26) gives

$$
\zeta_{\rho}^{\prime}(0)=-\frac{b^{2}}{4}(-2+\gamma+2 \ln 2)-b \zeta_{\mathrm{H}}^{\prime}\left(0, \frac{3}{2}+\frac{b}{2}\right)
$$




$$
\begin{aligned}
& +2\left[\zeta_{\mathrm{H}}\left(-1, \frac{3}{2}+\frac{b}{2}\right)+\zeta_{\mathrm{H}}^{\prime}\left(-1, \frac{3}{2}+\frac{b}{2}\right)\right]-2\left[\zeta_{\mathrm{H}}\left(-1, \frac{3}{2}\right)+\zeta_{\mathrm{H}}^{\prime}\left(-1, \frac{3}{2}\right)\right] \\
& +2\left\{\frac{1}{4} \ln \frac{c_{2}}{c_{1}}+\frac{11}{24} \ln \left[\frac{\chi^{\rho} c_{1}+c_{2}}{\sqrt{c_{1} c_{2}}\left(\chi^{\rho}+1\right)}\right]+\frac{b}{2}-\frac{b^{2}}{8}(2-\ln R-\gamma-2 \ln 2)\right. \\
& \left.-\int_{0}^{\infty} d k \ln k \frac{d}{d k} D_{2}\right\}, \quad b=\frac{\chi^{\rho} c_{1}-c_{2}}{\chi^{\rho} c_{1}+c_{2}} .
\end{aligned}
$$

In the case of nonmagnetic media $\left(\mu_{1}=\mu_{2}=1\right)$ the right-hand side of Eq. (A31) is slightly simplified. Assuming that we are dealing with a dilute dielectric ball we can expand $\zeta^{\prime}(0)$ in powers of the difference $\left(c_{1}-c_{2}\right)$, where $c_{1}=1 / \sqrt{\varepsilon_{1}}, \quad c_{2}=1 / \sqrt{\varepsilon_{2}}$. As a result we get

$$
\zeta^{\prime}(0)=\zeta_{\rho=-1}^{\prime}(0)+\zeta_{\rho=1}^{\prime}(0)=\frac{1}{4}\left(-\frac{7}{8}+\ln \frac{R}{c_{2}}+\ln 4+\gamma\right) \frac{\left(c_{1}-c_{2}\right)^{2}}{c_{2}^{2}}+\mathcal{O}\left(\left(c_{1}-c_{2}\right)^{3}\right) .
$$

\section{APPENDIX B: ZETA FUNCTION DETERMINANTS FOR ELECTROMAGNETIC FIELD WITH CYLINDRICALLY SYMMETRIC BOUNDARY CONDITIONS}

\section{A perfectly conducting cylindrical shell}

A complete spectral zeta function in the problem at hand is defined by the following expression [18]

$$
\begin{aligned}
\zeta(s)= & \frac{R^{2 s-1}}{2 \sqrt{\pi} c^{2 s} \Gamma(s) \Gamma(3 / 2-s)} \int_{0}^{\infty} d y y^{1-2 s} \frac{d}{d y} \ln \left[1-\mu_{0}^{2}(y)\right] \\
& +\frac{R^{2 s-1}}{\sqrt{\pi} c^{2 s} \Gamma(s) \Gamma(3 / 2-s)} \sum_{n=1}^{\infty} n^{1-2 s} \int_{0}^{\infty} d y y^{1-2 s} \frac{d}{d y} \ln \left[1-\mu_{n}^{2}(n y)\right],
\end{aligned}
$$

where

$$
\mu_{n}(y)=y \frac{d}{d y}\left[I_{n}(y) K_{n}(y)\right]
$$

The first term on the right hand side of Eq. (B1) is an analytic function of the complex variable $s$ in the strip $-1 / 2<\Re s<1 / 2$. Therefore there is no need in analytic continuation of this expression when calculating $\zeta^{\prime}(0)$. As regard to the second term in Eq. (B1) its analytic continuation to the region $\Re s<0$ can be accomplished in a standard way. We add and subtract here the uniform asymptotics of the integrand when $n$ tends to infinity

$$
\ln \left[1-\mu_{n}^{2}(n y)\right] \simeq-\frac{y^{4} t^{6}(y)}{4 n^{2}}+\mathcal{O}\left(n^{-4}\right), \quad t(y)=\frac{1}{\sqrt{1+y^{2}}}
$$

As a result we obtain 


$$
\begin{aligned}
\zeta(s)= & \frac{R^{2 s-1}}{2 \sqrt{\pi} c^{2 s} \Gamma(s) \Gamma(3 / 2-s)} \int_{0}^{\infty} \frac{d y}{y^{2 s-1}} \frac{d}{d y} \ln \left[1-\mu_{0}^{2}(y)\right] \\
& +\frac{R^{2 s-1}}{\sqrt{\pi} c^{2 s} \Gamma(s) \Gamma(3 / 2-s)} \sum_{n=1}^{\infty} n^{1-2 s} \int_{0}^{\infty} \frac{d y}{y^{2 s-1}} \frac{d}{d y}\left\{\ln \left[1-\mu_{n}^{2}(n y)\right]+\frac{y^{4} t^{6}}{4 n^{2}}\right\} \\
& -\frac{R^{2 s-1}}{32 \sqrt{\pi} c^{2 s}}(1-2 s)(3-2 s) \zeta_{\mathrm{R}}(2 s+1) \frac{\Gamma(1 / 2+s)}{\Gamma(s)} .
\end{aligned}
$$

Keeping in mind the behavior of the gamma function at the origin $\Gamma(s) \simeq s^{-1}$ one can easily find the derivative of $\zeta(s)$ at the point $s=0$

$$
\begin{aligned}
& \zeta^{\prime}(0)=\frac{1}{\pi R} \int_{0}^{\infty} d y y \frac{d}{d y} \ln \left[1-\mu_{0}^{2}(y)\right] \\
& +\frac{2}{\pi R} \sum_{n=1}^{\infty} n \int_{0}^{\infty} d y y \frac{d}{d y}\left\{\ln \left[1-\mu_{n}^{2}(n y)\right]+\frac{y^{4} t^{6}}{4 n^{2}}\right\}+\frac{1}{32 R}\left(3 \gamma-4-3 \ln \frac{2 c}{R}\right)
\end{aligned}
$$

Unlike the spherically symmetric boundaries, the integration is not removed in the formula obtained for $\zeta^{\prime}(0)$. Therefore the first two terms in Eq. (B4) can be calculated only numerically

$$
-\frac{1}{\pi R} \int_{0}^{\infty} d y \ln \left[1-\mu_{0}^{2}(y)\right]=\frac{0.53490}{R}
$$

Applying the FORTRAN subroutine that approximates the Bessel functions by Chebyshev's polynomials we evaluate the first 30 terms in the sum in Eq. (B4)

$$
-\frac{2}{\pi R} \sum_{n=1}^{\infty} n \int_{0}^{\infty} d y\left\{\ln \left[1-\mu_{n}^{2}(n y)\right]+\frac{y^{4} t^{6}}{4 n^{2}}\right\}=-\frac{0.00554}{R} .
$$

Finally gathering together all these results we have

$$
\zeta^{\prime}(0)=\frac{0.45847}{R}+\frac{3}{32 R} \ln \frac{R}{2 c} .
$$

\section{A compact infinite cylinder with $c_{1}=c_{2}$}

Now we turn to a compact cylinder placed into unbounded medium such that the velocity of light is uniform on the lateral surface of the cylinder. Proceeding as in the case of a cylindrical shell we start with the expression for the relevant spectral zeta function

$$
\zeta(s)=\frac{R^{2 s-1}}{2 \sqrt{\pi} c^{2 s} \Gamma(s) \Gamma(3 / 2-s)} \sum_{n=-\infty}^{\infty} \int_{0}^{\infty} d y y^{1-2 s} \frac{d}{d y} \ln \left[1-\xi^{2} \mu_{n}^{2}(y)\right]
$$

with parameter $\xi$ determined in Eq. (4.15). In the linear approximation with respect to $\xi^{2}$ Eq. (B8) assumes the form 


$$
\begin{aligned}
\zeta(s)= & -\frac{R^{2 s-1} \xi^{2}}{2 \sqrt{\pi} c^{2 s} \Gamma(s) \Gamma(3 / 2-s)} \int_{0}^{\infty} d y y^{1-2 s} \frac{d}{d y} \mu_{0}^{2}(y) \\
& -\frac{R^{2 s-1} \xi^{2}}{\sqrt{\pi} c^{2 s} \Gamma(s) \Gamma(3 / 2-s)} \sum_{n=1}^{\infty} \int_{0}^{\infty} d y y^{1-2 s} \frac{d}{d y} \mu_{n}^{2}(y) .
\end{aligned}
$$

The analytic continuation to the region $\Re s<0$ is needed only for the second term in Eq. (B9). Adding and subtracting here the uniform asymptotics of the integrand for large $n$

$$
-\mu_{n}^{2}(n y) \simeq-\frac{y^{4} t^{6}(y)}{4 n^{2}}+\mathcal{O}\left(n^{-4}\right)
$$

we obtain

$$
\begin{aligned}
\zeta^{\prime}(0)= & \left.-\frac{\xi^{2}}{\pi R} \int_{0}^{\infty} d y y \frac{d}{d y} \mu_{0}^{2}(y)+\frac{2 \xi^{2}}{\pi R} \sum_{n=1}^{\infty} n \int_{0}^{\infty} d y y \frac{d}{d y}\left[-\mu_{n}^{2}(n y)\right)+\frac{y^{4} t^{6}}{4 n^{2}}\right] \\
& +\frac{\xi^{2}}{32 R}\left(3 \gamma-4-3 \ln \frac{2 c}{R}\right) .
\end{aligned}
$$

The first two terms in Eq. (B11) can again be calculated only numerically

$$
\begin{gathered}
\frac{\xi^{2}}{\pi R} \int_{0}^{\infty} d y \mu_{0}^{2}(y)=\frac{\xi^{2}}{R} 0.28428 \\
-\frac{2 \xi^{2}}{\pi R} \sum_{n=1}^{\infty} n \int_{0}^{\infty} d y\left[-\mu_{n}^{2}(n y)+\frac{y^{2} t^{6}}{4 n^{2}}\right]=-\frac{\xi^{2}}{R} 0.00640 .
\end{gathered}
$$

The final result reads

$$
\begin{aligned}
\zeta^{\prime}(0) & =\frac{\xi^{2}}{R}\left[0.28428-0.00640+\frac{1}{32}\left(3 \gamma-4-3 \ln \frac{2 c}{R}\right)\right] \\
& =\frac{\xi^{2}}{R}\left(0.20699+\frac{3}{32} \ln \frac{R}{2 c}\right) .
\end{aligned}
$$




\section{REFERENCES}

[1] B. W. Harris, F. Chen, and U. Mohideen, Phys. Rev. A 62, 052109/1-5 (2000).

[2] A. Roy, Lin Chiung-Yuan, and U. Mohideen, Phys. Rev. D 60, 111101/1-5 (1999).

[3] A. Roy and U. Mohideen, Phys. Rev. Lett. 82, 4380 (1999).

[4] M. J. Sparnaay, Physica 24, 751 (1958).

[5] J. Mehra, Physica 37, 145 (1967).

[6] J. S. Dowker and G. Kennedy, J. Phys. A 11, 895 (1978).

[7] K. Kirsten, J. Phys. A 24, 3281 (1991).

[8] K. Kirsten, Class. Quantum Grav. 8, 2239 (1991); 10, 1461 (1993).

[9] K. Kirsten, J. Math. Phys. 32, 3008 (1991).

[10] P. B. Gilkey, Invariance Theory, The Heat Equation and the Atiyah-Singer Index Theorem, 2nd. Edn. (CTC Press, Boca Raton, 1995).

[11] I. Avramidi, Heat Kernel and Quantum Gravity (Lecture Notes in Physics. m54) (Springer, Berlin, 2000).

[12] M. Bordag, U. Mohideen, and V. M. Mostepanenko, Phys. Rep. 353, No. 1-3, 1-205 (2001).

[13] J. Feinberg, A. Mann, and M. Revzen, Ann. Phys. (N.Y.) 288, 103 (2001).

[14] R. Balian and B. D. Duplantier, Ann. Phys. (N.Y.) 104, 300 (1977); 112, 165 (1978).

[15] M. Bordag, D. Vassilevich, H. Falomir, and E. M. Santangelo, Phys. Rev. D 64, 045017 (2001).

[16] J. Ambjørn and S. Wolfram, Ann. Phys. (N.Y.) 147, 1, 33 (1983).

[17] E. Elizalde, Ten physical applications of spectral zeta functions (Springer, Berlin, 1995).

[18] G. Lambiase, V. V. Nesterenko, and M. Bordag, J. Math. Phys. 40, 6254 (1999).

[19] M. Bordag and I. G. Pirozhenko, Phys. Rev. D 64, 025019 (2001).

[20] M. Bordag, B. Geyer, K. Kirsten, and E. Elizalde, Commun. Math. Phys. 179, 215 (1996).

[21] M. Bordag, K. Kirsten, and D. Vassilevich, Phys. Rev. D 59, 085011 (1999).

[22] E. Borgnis and C. H. Papas, "Electromagnetic Waveguides and Resonators," In Encyclopedia of Physics, Ed. S. Flüge (Springer Verlag, Berlin, 1958) v. XVI, pp. 285-422.

[23] B. S. DeWitt, Phys. Rep. 19, 295 (1975).

[24] G. Plunien, B. Muller, W. Greiner, Phys. Rep. 134, 87 (1986).

[25] M. Bordag, K. Kirsten, E. Elizalde, J. Math. Phys. 37 (1996) 895.

[26] J. A. Stratton, Electromagnetic Theory (McGraw-Hill, New York, 1941).

[27] Eur. Phys. J. C 15, No. 1-4, 73 (2000).

[28] I. Brevik, Phys. Rep. 52, 133 (1979).

[29] I. Brevik, V. V. Nesterenko, and I. G. Pirozhenko, J. Phys. A 31, 8661 (1998).

[30] V. V. Nesterenko, G. Lambiase, and G. Scarpetta, Phys. Rev. D 64, 025013 (2001).

[31] I. Klich, J. Feinberg, A. Mann, and M. Revzen, Phys. Rev. D 62, 045017 (2000).

[32] M. Bordag, V. V. Nesterenko, and I. G. Pirozhenko, "High temperature asymptotics in terms of heat kernel coefficients: boundary conditions with spherical and cylindrical symmetries", to appear in Nucl.Phys.Proc.Suppl.104:228-231,2002.

[33] G. Barton, "Perturbative Casimir shifts of spheres: towards re-orienting and an agenda". Talk at the Fifth Workshop on Quantum Field Theory Under the Influence of External Conditions (Leipzig, September 10 - 14, 2001). 
[34] G. Barton, J. Phys. A 34, 4083 (2001).

[35] G. Barton, Phys. Rev. A 64, 032103 (2001).

[36] L. L. DeRaad, Jr. and K. Milton, Ann. Phys. (N.Y.) 136, 229 (1981).

[37] K. A. Milton, A. V. Nesterenko, and V. V. Nesterenko, Phys. Rev. D 59, 105009 (1999).

[38] V. V. Nesterenko and I. G. Pirozhenko, Phys. Rev. D 60, 125007 (1999).

[39] P. Gosdzinski and A. Romeo, Phys. Lett. B 441, 265 (1998).

[40] M. L. Levin, S. M. Rytov, Theory of Equilibrium Thermal Fluctuations in Electrodynamics [in Russian] (Nauka, Moscow, 1967).

[41] I. S. Gradshteyn and I. M. Ryzhik, Table of Integrals, Series and Products, Fifth Edition (Academic Press, New York, 1994). 